\title{
Age-Related Changes in Instrumental and Basic Activities of Daily Living Impairment in Older Adults with Very Mild Alzheimer's Disease
}

\author{
Takayuki Tabira $^{a}$ Maki Hottab Miki Muratac Kazuhiro Yoshiurad \\ Gwanghee Han $^{d}$ Tomohisa Ishikawa ${ }^{e}$ Asuka Koyamad Noriyuki Ogawa $^{f}$ \\ Michio Maruta ${ }^{a}$ Yuriko Ikeda ${ }^{a}$ Takaaki Morig Taku Yoshidag \\ Mamoru Hashimotoh ${ }^{h}$ Manabu Ikedab \\ ${ }^{a}$ Department of Clinical Neuropsychiatry, Graduate School of Health Sciences, Kagoshima \\ University, Kagoshima, Japan; ${ }^{b}$ Department of Psychiatry, Graduate School of Medicine, \\ Osaka University, Osaka, Japan; ' ${ }^{\circ}$ epartment of Occupational Therapy, Kumamoto Seimei \\ Hospital, Kumamoto Seimei, Japan; d Department of Neuropsychiatry, Faculty of Life \\ Sciences, Kumamoto University, Kumamoto, Japan; e Department of Neuropsychiatry,

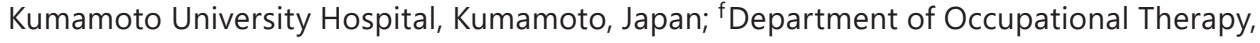 \\ Faculty of Health Sciences, Kyoto Tachibana University, Kyoto, Japan; 9 Department of \\ Neuropsychiatry, Molecules and Function, Ehime University Graduate School of Medicine, \\ Ehime, Japan; h'Department of Behavioral Neurology and Neuropsychiatry, Osaka University, \\ Osaka, Japan
}

\section{Keywords}

Aging · Very mild Alzheimer's disease · Activities of daily living

\begin{abstract}
Background/Aims: Age-related changes in impairments in activities of daily living (ADL) in older adults with very mild Alzheimer's disease (vmAD) have been scarcely explored. We clarified the characteristics of ADL impairment and examined how ADL impairments differed by age in such patients compared with community-dwelling cognitively normal older adults. Methods: The participants were 107 older adults with vmAD (Mini-Mental State Examination [MMSE] score 224 ), all of whom were first-visit outpatients at the Dementia Clinic of the Department of Neuropsychiatry, Kumamoto University Hospital. The controls were 682 community-dwelling older adults who participated in the 3rd Nakayama Study with MMSE score $\geq 24$. We examined the association of instrumental and basic ADL (IADL and BADL, respectively) independence with the odds of vmAD using multiple logistic regression analysis and determined differences in ADL impairment by age using age- and sex-matched analysis. Results:
\end{abstract}


Impairments in handling finances (OR 57.08), managing medication (OR 5.13), and dressing (OR 3.35; BADL) were associated with greater odds of vmAD. Among those aged 65 years and above, there were fewer patients with vmAD than healthy controls who could independently handle finances and medication. Among patients with vmAD, the percentages of those who could independently manage shopping, food preparation, and housekeeping only decreased after age 74. Age-related decreases in independence were observed in few BADL items; these, however, were temporary. Conclusions: Patients with vmAD show significantly decreased IADL independence from early old age.

(C) 2020 The Author(s)

Published by S. Karger AG, Basel

\section{Introduction}

Aging is associated with decreases in the degree of independence in instrumental and basic activities of daily living (IADL and BADL, respectively) in community-dwelling older adults. Impairments in IADL, such as using public transportation, shopping, preparing meals, paying bills, and managing deposits, tend to appear earlier than impairments in BADL (e.g., transferring or bathing) among normally aging individuals [1-3].

Patients with mild cognitive impairment (MCI) and very mild Alzheimer's disease (vmAD) also tend to show declines in complex IADL associated with cognitive function. IADL impairment is a predictive factor for dementia, along with advanced age and a low score on the Mini-Mental State Examination (MMSE) [4]. Among the IADL in patients with MCI and mild AD, the items showing the greatest impairment were shopping, food preparation, transportation use, and handling medications and finances [5-7]. As per a systematic review, the item that showed impairment across the included studies was handling finances [8]. According to the Diagnostic and Statistical Manual of Mental Disorders, Fifth Edition, mild neurocognitive disorder is defined by impairments in complex IADL such as bill payment and medication management [9].

Classification of $\mathrm{MCI}$ and $\mathrm{AD}$ among older adults is based on not only cognitive function but also the degree of decrease in IADL/BADL independence. However, as noted above, IADL/ BADL independence also decreases with normal aging. Thus, it remains unclear whether agerelated changes in IADL/BADL among patients with vmAD show a similar tendency to those among healthy older adults. It is necessary to clarify this point to correctly diagnose MCI and vmAD, regardless of age, and thereby move toward the development of preventive interventions. The purpose of this study was, therefore, to clarify the characteristics of IADL/BADL impairment in patients with vmAD and how these characteristics differ according to age in comparison with community-dwelling normal older adults.

\section{Methods}

\section{Participants}

The participants were first-visit outpatients at the Dementia Clinic of the Department of Neuropsychiatry, Kumamoto University Hospital, who were recruited at the clinic between April 2007 and 2014. The inclusion criteria were as follows: (1) diagnosis of AD, (2) MMSE score $\geq 24$, (3) living at home, (4) had enough information providers (e.g., caregiver), and (5) aged 65 years or older. The exclusion criteria were physical and serious psychiatric diseases that prominently affect activities of daily living (ADL) independence. Overall, 107 patients with vmAD were recruited (Table 1). All the patients were diagnosed with probable AD according to the National Institute for Neurological and Communicative Disorders and Stroke 
Table 1. Characteristics of the participants

\begin{tabular}{|c|c|c|c|c|c|c|}
\hline \multirow[t]{2}{*}{ Variable } & \multicolumn{3}{|c|}{ Before matching } & \multicolumn{3}{|c|}{ After matching } \\
\hline & $\begin{array}{l}\text { vmAD } \\
(n=107)\end{array}$ & $\begin{array}{l}\mathrm{HC} \\
(n=682)\end{array}$ & $p$ value $^{\mathrm{a}}$ & $\begin{array}{l}\text { vmAD } \\
(n=104)\end{array}$ & $\begin{array}{l}\mathrm{HC} \\
(n=104)\end{array}$ & $p$ value $^{\mathrm{a}}$ \\
\hline Age, years, mean \pm SD & $78.1 \pm 6.0$ & $73.7 \pm 5.5$ & $<0.001$ & $77.9 \pm 5.9$ & $77.9 \pm 5.9$ & 1.000 \\
\hline Women, $n(\%)$ & $58(54.2)$ & $375(54.7)$ & 0.708 & $57(54.8)$ & $57(54.8)$ & 1.000 \\
\hline Education, years, mean \pm SD & $11.6 \pm 2.7$ & $11.0 \pm 3.8$ & 0.456 & $11.6 \pm 2.7$ & $11.2 \pm 3.3$ & 0.532 \\
\hline MMSE, mean \pm SD & $25.4 \pm 1.5$ & $28.0 \pm 1.9$ & $<0.001$ & $25.6 \pm 1.5$ & $26.0 \pm 1.9$ & 0.008 \\
\hline PSMS, mean \pm SD & $5.5 \pm 0.8$ & $5.9 \pm 0.7$ & $<0.001$ & $5.5 \pm 0.8$ & $5.7 \pm 1.0$ & 0.362 \\
\hline \multicolumn{7}{|l|}{ Lawton IADL, women } \\
\hline (max 8), mean \pm SD & $6.3 \pm 1.4$ & $7.8 \pm 0.7$ & $<0.001$ & $6.3 \pm 1.5$ & $7.6 \pm 0.9$ & $<0.001$ \\
\hline \multicolumn{7}{|l|}{ Lawton IADL, men } \\
\hline$(\max 5)^{\mathrm{b}}$, mean $\pm \mathrm{SD}$ & $3.8 \pm 1.1$ & $4.9 \pm 0.6$ & $<0.001$ & $3.8 \pm 1.2$ & $4.7 \pm 0.9$ & $<0.001$ \\
\hline
\end{tabular}

HC, healthy control; vmAD, very mild Alzheimer's disease; MMSE, Mini-Mental State Examination; PSMS, Physical SelfMaintenance Scale; IADL, instrumental and basic activities of daily living.

a Student $t$ test for continuous measures and Pearson $\chi^{2}$ test for proportions.

${ }^{\mathrm{b}}$ Lawton IADL scores for men excluded 3 items (preparing food, housekeeping, and doing laundry).

and the AD and Related Disorders Association [10]. Comparative controls were 1,290 community-dwelling older adults who participated in the 3rd Nakayama Study (Ehime, Japan) [11]. Six hundred and eighty-two older people fulfilled the inclusion criteria of an MMSE score $\geq 24$ and being aged 65 years or older; they were designated as the healthy control (HC) group. Exclusion criteria were (1) diagnosis of dementia and (2) physical diseases, such as stroke, that prominently affect ADL independence. The diagnosis of dementia was established according to DSMIII-R criteria. Both vmAD and HC were designated by senior neuropsychiatrists. This study was approved by the Human Ethics Review Committee of Kumamoto University. Written informed consent was obtained from all patients and their primary caregivers (all family members).

\section{Measures}

The MMSE, Physical Self-Maintenance Scale (PSMS), and Lawton IADL Scale were administered by senior neuropsychiatrists, clinical psychologists, and occupational therapists with adequate experience in caring for and rehabilitation of patients with dementia. The MMSE is one of the most frequently used cognitive screening questionnaires $[12,13]$. The PSMS is used to evaluate patients' ability to perform BADL $[14,15]$. It contains 6 items, assessing independence in toileting, feeding, dressing, grooming, physical ambulation, and bathing. Scores range from 0 to 6, with higher scores indicating better functioning. The Lawton IADL Scale evaluates 8 domains of function: ability to use the telephone, shopping, food preparation, housekeeping, laundry, mode of transportation, responsibility for own medication, and ability to handle finances $[14,15]$. This questionnaire was completed using information provided by the caregivers. Scores range from 0 to 8 in women (all items) and 0-5 in men (as food preparation, housekeeping, and laundry are excluded) [14].

\section{Statistical Analysis}

First, Student $t$ tests and Pearson's $\chi^{2}$ tests were used to identify differences in characteristics between the vmAD and HC groups. Second, multiple logistic regression analysis was used to explore to what extent impairment in each IADL and BADL item was associated with vmAD compared to the HC group. The independent variables were the degree of indepen- 
dence in each PSMS item (e.g., toileting, grade 1: can appropriately use the toilet by oneself and exhibits no incontinence; grade 2: needs to be reminded to use the toilet, needs help in cleaning self, and has a rare accident; grade 3: soils or wets oneself while asleep more than once a week; grade 4: soils or wets oneself while awake more than once a week; grade 5: no control over bowels or bladder) and Lawton IADL Scale item (e.g., use of telephone, grade 1: operates telephone on own initiative, looks up and dials numbers; grade 2: can dial a few wellknown numbers; grade 3: can answer telephone, but not dial; grade 4: does not use a telephone at all). Age and MMSE score were controlled for in this analysis.

Finally, we divided participants into age groups in 3-year intervals and calculated the percentage of independent persons (grade 1 on the PSMS and Lawton IADL Scale items) in each age group to examine age-related changes. We employed propensity score matching to remove age and sex biases for comparison between the vmAD and HC groups. We used logistic regression analysis to calculate the propensity scores and performed one-to-one age and sex matching without replacement. Subsequently, Pearson's $\chi^{2}$ tests were used to examine differences in the percentages of independent persons (independence ratios) for all items between the vmAD and $\mathrm{HC}$ groups in each age group. A moving average of 3 points was used to display the age-related changes [16].

All analyses were conducted using IBM SPSS Statistics 24.0 (IBM Japan Tokyo, Japan). The statistical significance level was set at $p<0.05$.

\section{Results}

\section{Participant Characteristics}

Participants' characteristics and the comparisons between the vmAD and HC groups, before and after age matching, are shown in Table 1 . Before age matching, patients with vmAD were significantly older (vmAD $78.1 \pm 6.0$, HC $73.7 \pm 5.5, p<0.0001$ ) and had significantly lower MMSE scores (vmAD $25.4 \pm 1.5$, HC $28.0 \pm 1.9, p<0.001$ ) compared to the HC group. Moreover, patients with vmAD had slightly lower PSMS scores (vmAD 5.5 \pm 0.8 , HC 5.9 \pm 0.7 , $p<0.001$ ) and significantly lower Lawton IADL Scale scores (women: vmAD 6.3 \pm 1.4 , HC 7.8 $\pm 0.7, p<0.001$, men: vmAD $3.8 \pm 1.1$, HC $4.9 \pm 0.6, p<0.001$ ) than the HC group. There were no differences in sex distribution and educational levels between the 2 groups.

Participants' characteristics after age and sex matching are depicted in Table 1. Patients with vmAD were significantly older (vmAD $78.1 \pm 6.0$, HC $73.7 \pm 5.5, p<0.0001$ ) and had significantly lower MMSE scores (vmAD $25.4 \pm 1.5$, HC $28.0 \pm 1.9, p<0.001$ ). Among patients with vmAD, PSMS scores were slightly lower (vmAD $5.5 \pm 0.8$, HC $5.9 \pm 0.7, p<0.001$ ), whereas Lawton IADL Scale scores were significantly lower (women: vmAD $6.3 \pm 1.4$, HC 7.8 $\pm 0.7, p<$ 0.001 , men: vmAD $3.8 \pm 1.1$, HC $4.9 \pm 0.6, p<0.001$ ) than among the HC group. The MMSE, PSMS, and Lawton IADL Scale scores in the vmAD group were significantly lower than in the HC group, even after age and sex matching.

\section{IADL/BADL Independence in Patients with vmAD}

Table 2 depicts the items showing IADL/BADL impairment that distinguish the vmAD and HC groups. IADL impairments significantly associated with the odds of having vmAD were handling finances (OR 57.08, 95\% CI 9.93-327.87, $p<0.001$ ) and handling medications (OR 5.13, 95\% CI 1.19-22.07, $p=0.028$ ). Therefore, lower independence in handling finances and medications was associated with greater odds of being in the vmAD group.

As for the BADL items, only lower independence in dressing (OR 3.35, 95\% CI 1.41-7.93, $p=0.006$ ) was associated with increased odds of vmAD. 
Table 2. IADL/BADL independence in VmAD patients

a. ORs for vmAD patients in IADL item

\begin{tabular}{|c|c|c|c|c|c|}
\hline & $\mathrm{B}(\mathrm{SE})$ & Wald & OR & $95 \% \mathrm{CI}$ & $p$ value \\
\hline \multicolumn{6}{|l|}{ Lawton IADL } \\
\hline Using the telephone & $-0.99(0.78)$ & 1.62 & 0.37 & $0.08-1.71$ & 0.204 \\
\hline Shopping & $0.56(0.61)$ & 0.86 & 1.75 & $0.53-5.74$ & 0.355 \\
\hline Preparing food & $0.16(0.31)$ & 0.27 & 1.18 & $0.64-2.17$ & 0.601 \\
\hline Housekeeping & $0.08(0.40)$ & 0.05 & 1.09 & $0.49-2.38$ & 0.831 \\
\hline Laundry & $-0.97(0.81)$ & 3.93 & 0.24 & $0.18-1.08$ & 0.055 \\
\hline Using transportation & $-0.56(0.47)$ & 1.45 & 0.57 & $0.22-1.42$ & 0.229 \\
\hline Handling medications & $1.63(0.89)$ & 4.81 & 5.13 & $1.19-22.07$ & 0.028 \\
\hline Handling finances & $4.04(0.89)$ & 20.55 & 57.08 & $9.93-327.87$ & $<0.001$ \\
\hline Age, years & $0.11(0.04)$ & 7.84 & 1.12 & $0.81-1.01$ & 0.005 \\
\hline MMSE & $-0.62(0.13)$ & 22.65 & 0.53 & $1.45-2.48$ & $<0.001$ \\
\hline$R^{2}$ & 0.70 & & & & \\
\hline \multicolumn{6}{|c|}{ b. ORs for vmAD patients in BADL item } \\
\hline & $\mathrm{B}(\mathrm{SE})$ & Wald & OR & $95 \% \mathrm{CI}$ & $p$ value \\
\hline \multicolumn{6}{|l|}{ PSMS } \\
\hline Toileting & $0.11(035)$ & 0.10 & 1.11 & $0.56-2.26$ & 0.752 \\
\hline Feeding & $-0.27(0.90)$ & 0.09 & 0.76 & $0.13-4.45$ & 0.762 \\
\hline Dressing & $1.21(0.44)$ & 7.57 & 3.35 & $1.41-7.93$ & 0.006 \\
\hline Grooming & $0.02(0.59)$ & 0.01 & 1.02 & $0.32-3.24$ & 0.973 \\
\hline Locomotion & $-0.16(0.27)$ & 0.34 & 0.85 & $0.49-1.45$ & 0.557 \\
\hline Bathing & $-1.2(0.63) 1$ & 3.68 & 0.29 & $0.85-1.03$ & 0.055 \\
\hline Age, years & $0.11(0.02)$ & 27.62 & 1.11 & $1.07-1.16$ & $<0.001$ \\
\hline MMSE & $-0.67(0.07)$ & 88.41 & 0.51 & $0.44-0.58$ & $<0.001$ \\
\hline$R^{2}$ & 0.42 & & & & \\
\hline
\end{tabular}

IADL, instrumental activities of daily living; BADL, basic activities of daily living; PSMS, Physical SelfMaintenance Scale, vmAD, very mild Alzheimer's disease; MMSE, Mini-Mental State Examination.

Impairment in IADL/BADL Items According to Age

The independence ratios for the BADL and IADL items in each age group are depicted in Table 3 and Figures 1 and 2. As seen in Figures 1 and 2, both groups showed decreases in BADL and IADL independence with aging; however, the decreasing slope and items differed between patients with vmAD and the HC group. As for the IADL items, the independence ratios of handling medication and finances among patients with vmAD were significantly lower than the HC group in all age groups between 65 and 88 years $(p<0.01)$. For those aged over 74 years, the independence ratios of shopping $(p<0.01)$, preparing food $(p<0.05)$, and housekeeping $(p<0.01)$ were significantly lower among patients with vmAD than among HC individuals, while for those aged 77 years and above, the independence ratios for using the telephone $(p<0.01)$ and transportation $(p<0.01)$ were significantly lower among patients with vmAD than among HC individuals. The independence ratio of doing laundry did not significantly decrease with aging. As for the BADL items, the independence ratios of locomotion were significantly lower among patients with vmAD than among HC individuals after age $77(p<0.05)$, while those for dressing were lower after age $80(p<0.05)$. No other significant differences were observed. 


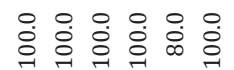

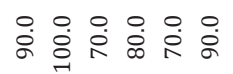

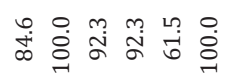

+艹

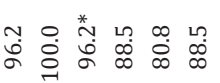

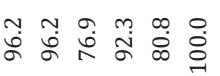

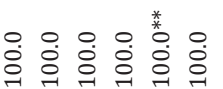

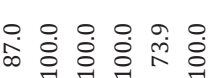

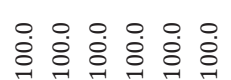

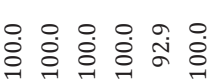

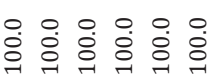

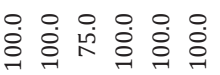

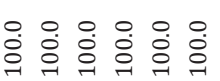

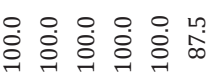

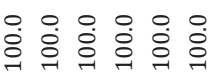

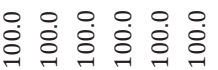

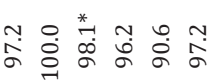

岁

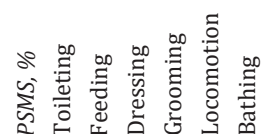

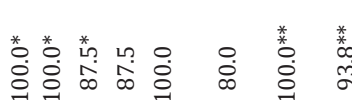

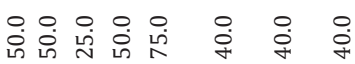

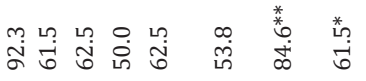

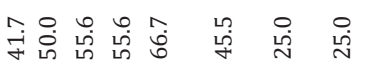

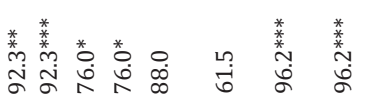

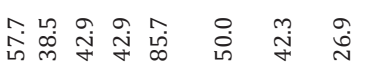

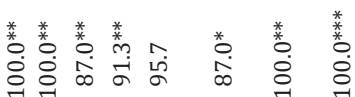

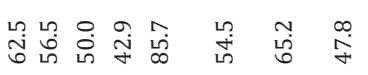

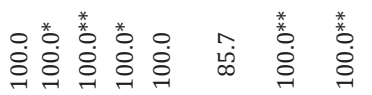

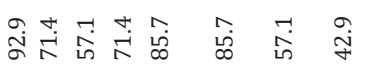

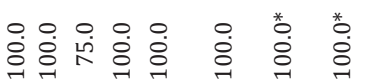

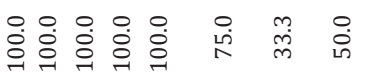

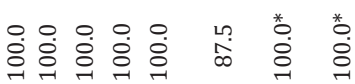

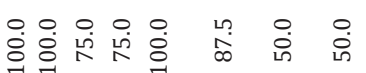

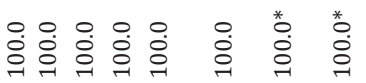

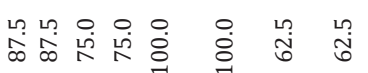

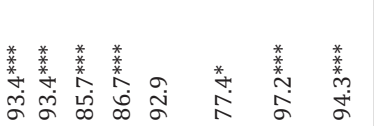

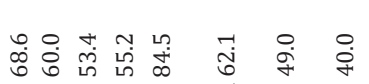

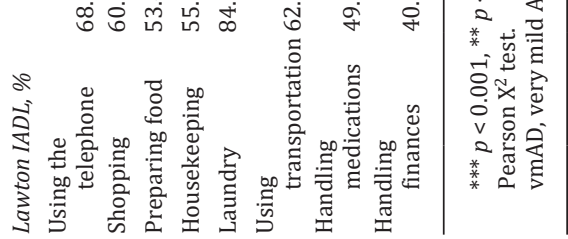




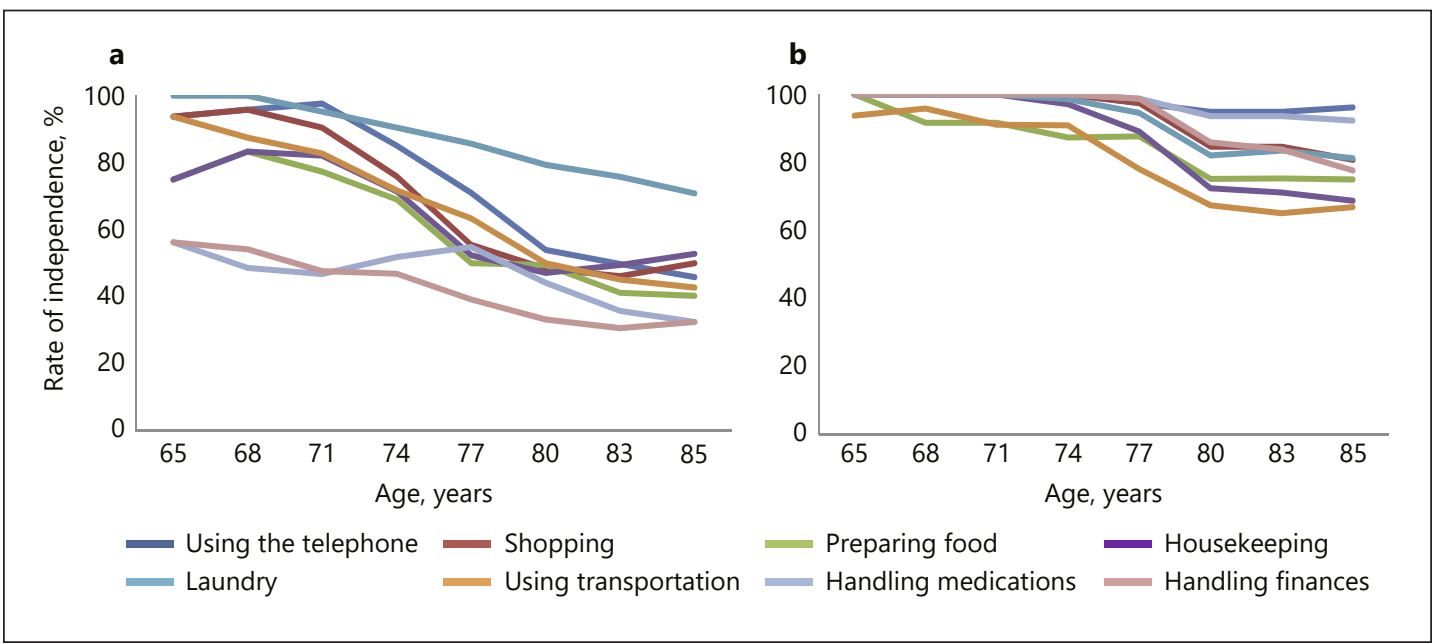

Fig. 1. a, b Items of IADL independence with aging in Lawton IADL. Percentage of independent persons (grade 1 on the Lawton IADL Scale items) in each age group. While both groups generally showed decreases in IADL independence with aging, the decreasing slope was steeper in patients with vmAD compared to HC. a Very mild AD patients $(n=104)$. b Healthy controls $(n=104)$.

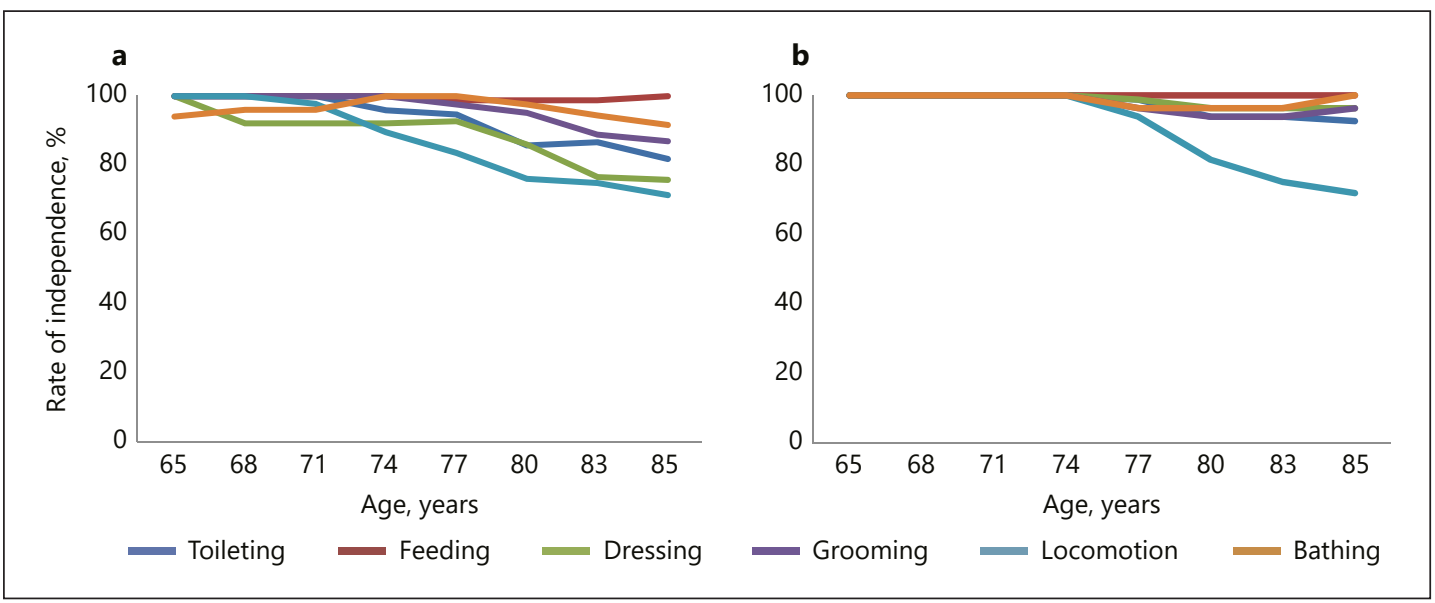

Fig. 2. a, b Items of BADL independence with aging in PSMS. Percentage of independent persons (grade 1 on the PSMS items) in each age group. a Very mild AD patients $(n=104)$. b Healthy control $(n=104)$.

\section{Discussion}

In this study, we examined (1) which IADL and BADL impairments were associated with vmAD using multiple logistic regression analysis and (2) which IADL and BADL impairments in each age group differed between patients with vmAD and $\mathrm{HC}$ individuals using propensity score matching.

IADL/BADL Independence in Patients with vmAD

Among the IADL items, handling finances had the strongest association with vmAD (OR 57.08), followed by handling medication (OR 5.13). These findings coincide with those of past studies, wherein independence in using the telephone, medication and money management, 
using home appliances, and transport was significantly lower among patients with amnestic MCI than in HC individuals [5, 6]. Moreover, a systematic review of 35 articles on patients with MCI by Jekel et al. [8] revealed that the most common IADL impairment was in handling finances, followed by handling medication, using the telephone, and schedule keeping. Handling finances and medications are complex IADL requiring advanced cognitive functions such as executive function $[17,18]$ as well as self-management for life over time [19].

A score of 1 on the handling finances item of the Lawton IADL Scale [14], which mentions "is able to manage financial matters independently (e.g., budget, banking)" and "manages dayto-day purchases," indicated that individuals had sufficient cognitive function, including memory [20], visuospatial cognitive function, and executive function [21]. Moreover, a score of 1 on the handling medication item ("responsible for taking medication in the correct dosages at the correct time") suggested that participants had sufficient time orientation ability and could check the type and dosages of their medication. Independence in such complex IADL was lower in patients with vmAD because these individuals show deficits in various cognitive functions necessary for life management, including executive function [6], memory [20], and time orientation [22].

As for the BADL, only dressing (OR 3.35) showed a significant relationship with the odds of vmAD. Previous research has shown that declines in BADL independence in patients with $\mathrm{AD}$ are associated with an MMSE score of 17 [23] and Clinical Dementia Rating score of 1 [24]. In other words, BADL deficits begin only when cognitive decline progresses to the mild-tomoderate stage. Bathing, dressing, and grooming are particularly sensitive to declines after this stage [25], which is similar to our findings. Dressing might have shown a decline because it is thought to be related to visuospatial perception and body scheme [26] and may be impaired in the relatively mild stage of $\mathrm{AD}$ [27].

\section{IADL/BADL Independence According to Age}

As shown in Figure 1, while both groups generally showed decreases in IADL independence with aging, the decreasing slope was steeper in patients with vmAD compared to HC. The decrease in independence began from young-old age in patients with vmAD (Table 3). In particular, independence in handling medication and finances was significantly lower in patients with vmAD than in HC individuals from ages 65 to 88. In the 60s, patients with vmAD remained at 50-60\% independence, while all HC were independent. Patients with vmAD showed impairment in those items from an early age. In older people with subjective memory complaints, the "handling the right time to take medicine" and "keeping track of how much medicine remains" aspects of handling medication are impaired, while the "handling cash" and "understanding household expenses" aspects of handling finances are likely to be disturbed [28]. These activity processes in patients with vmAD may be impaired from an early age.

In addition, independence in shopping, preparing food, and housekeeping was significantly lower in individuals with vmAD than in HC from the age of 74 years. Specifically for the 74-76 age group, the independence ratios for these items for HC were $100 \%$, whereas those of patients with vmAD were $50-70 \%$. Shopping, preparing food, and housekeeping all require a level of cognitive functioning that is useful for daily living, as demonstrated by items such as "Takes care of all shopping needs independently," "Plans, prepares, and serves adequate meals independently," and "Maintains the house alone or with occasional assistance" [14]. Therefore, these items will show decreased independence in patients with vmAD with aging after impairments in handling medication and finances. In the age group of 77-82 years, independence in all IADL items, except for laundry, decreased significantly. IADL execution is required for activities such as operating tools and machines, managing goods, selecting tools, and monitoring [28]. People with vmAD may also experience difficulties in daily life activities including these elements, which require memory and executive functions. Therefore, even 
after matching for age and sex, IADL independence in patients with vmAD decreased significantly with age compared to the HC group.

Among the BADL items, there was a significant decrease in independence in locomotion in the 77-79 age group and in dressing in the 80-82 age group; no other BADL items showed declines compared to HC individuals. As shown in Figure 2, as only locomotion decreased after age 74 in $\mathrm{HC}$, locomotion and dressing were the only aspects to change greatly from an early age in patients with vmAD. In patients with vmAD with slight cognitive decline, the BADL impairments were very few even when age-related changes were considered. BADL impairments are likely to appear from mild to severe dementia [23]. It might be suggested that patients with vmAD displayed declines only in complex IADL independence. Thus, agerelated changes in ADL independence are different between patients with vmAD and HC individuals. The strengths of this study were (1) valuable data about vmAD patients with MMSE score $\geq 24$ was collected and (2) the age at which patients started their decline in each ADL independence area was found. This may be useful information in preventive rehabilitation and care for IADL impairment in patients with vmAD.

Several limitations should be noted. First, there were relatively few individuals in each age group, as we divided participants according to 3-year intervals and performed one-to-one age and sex matching in order to obtain detailed data on the influence of age on ADL in older people with or without AD. Second, the age-related changes in IADL/BADL were examined cross-sectionally, not longitudinally. Third, the possibility of selection bias cannot be ruled out because patients with vmAD were recruited from a memory clinic and HC from the community. Finally, older adults are likely to have multiple diseases, but we did not account for the possible effects of other diseases on ADL. However, our study has merit because of its novel focus on age-related changes in ADL in patients with vmAD. Future replication studies should include larger samples and consider other geriatric diseases to confirm our results.

\section{Conclusion}

In this study, we examined the IADL and BADL items associated with vmAD, and how IADL and BADL impairments differed according to age in patients with vmAD compared to community-dwelling cognitively normal older adults. Impairments in handling finances and medication (IADL) and dressing (BADL) were associated with the odds of vmAD. As for agerelated changes in $\mathrm{VmAD}$, we found that independence in handling finances and medication began to decline from age 65, while independence in shopping, preparing food, and housekeeping began to decline from age 74. Few BADL items declined with aging, and those that did began to show declines later on. Overall, IADL independence in patients with vmAD is significantly affected even from early older adulthood.

\section{Acknowledgment}

We appreciate the contribution of all our participants. We also thank support from president and occupational therapists in Ishikawa Prefectural Takamatsu Hospital.

\section{Statement of Ethics}

This study was approved by the Human Ethics Review Committee of Kumamoto University. Written informed consent was obtained from all the participants. 


\section{Disclosure Statement}

The authors have no conflicts of interest to declare.

\section{Funding Sources}

This work was supported by MHLW KAKENHI Grant Number 15654752, "Study on 30 standardization of rehabilitation for dementia based on analysis of daily activity impairment."

\section{Author Contributions}

T.T.: designed the study and wrote the initial draft of the manuscript. M.H., M.M., K.Y., T.I., A.K., T.M., T.Y., M.H., and M.I.: contributed to data collection, extraction and interpretation, and assisted in the preparation of the manuscript. All other authors contributed to the interpretation of data and critically reviewed the manuscript. All authors approved the final version of the manuscript and agreed to be accountable for all aspects of the work.

\section{References}

1 Manton KG, Land KC. Active life expectancy estimates for the U.S. elderly population: a multidimensional continuous-mixture model of functional change applied to completed cohorts, 1982-1996. Demography. 2000 Aug;37(3):253-65.

2 Ishizaki T, Kobayashi Y, Kai I. Functional transitions in instrumental activities of daily living among older Japanese. J Epidemiol. 2000 Jul;10(4):249-54.

3 Liang Y, Welmer AK, Wang R, Song A, Fratiglioni L, Qiu C. Trends in Incidence of Disability in Activities of Daily Living in Chinese Older Adults: 1993-2006. J Am Geriatr Soc. 2017 Feb;65(2):306-12.

4 Pérès K, Chrysostome V, Fabrigoule C, Orgogozo JM, Dartigues JF, Barberger-Gateau P. Restriction in complex activities of daily living in MCI: impact on outcome. Neurology. 2006 Aug;67(3):461-6.

5 Kim KR, Lee KS, Cheong HK, Eom JS, Oh BH, Hong CH. Characteristic profiles of instrumental activities of daily living in different subtypes of mild cognitive impairment. Dement Geriatr Cogn Disord. 2009;27(3):278-85.

6 Mariani E, Monastero R, Ercolani S, Rinaldi P, Mangialasche F, Costanzi E, et al.; ReGAl Study Group. Influence of comorbidity and cognitive status on instrumental activities of daily living in amnestic mild cognitive impairment: results from the ReGAl project. Int J Geriatr Psychiatry. 2008 May;23(5):523-30.

7 Hesseberg K, Bentzen H, Ranhoff AH, Engedal K, Bergland A. Disability in instrumental activities of daily living in elderly patients with mild cognitive impairment and Alzheimer's disease. Dement Geriatr Cogn Disord. 2013;36(3-4):146-53.

8 Jekel K, Damian M, Wattmo C, Hausner L, Bullock R, Connelly PJ, et al. Mild cognitive impairment and deficits in instrumental activities of daily living: a systematic review. Alzheimers Res Ther. 2015 Mar;7(1):17.

9 American Psychiatric Association. DSM-5: diagnostic and statistical manual of mental disorders. 5th ed. Washington (DC): American Psychiatric Publishing; 2013.

10 McKhann G, Drachman D, Folstein M, Katzman R, Price D, Stadlan EM. Clinical diagnosis of Alzheimer's disease: report of the NINCDS-ADRDA Work Group under the auspices of Department of Health and Human Services Task Force on Alzheimer's Disease. Neurology. 1984 Jul;34(7):939-44.

11 Matsumoto N, Ikeda M, Fukuhara R, Shinagawa S, Ishikawa T, Mori T, et al. Caregiver burden associated with behavioral and psychological symptoms of dementia in elderly people in the local community. Dement Geriatr Cogn Disord. 2007;23(4):219-24.

12 Folstein MF, Folstein SE, McHugh PR. "Mini-mental state". A practical method for grading the cognitive state of patients for the clinician. J Psychiatr Res. 1975 Nov;12(3):189-98.

13 Mori E, Mitani Y, Yamadori A. Usefulness of a Japanese version of the Mini-Mental State Test in neurological patients. Jpn J Neuropsychol. 1985;1:82-90.

14 Lawton MP, Brody EM. Assessment of older people: self-maintaining and instrumental activities of daily living. Gerontologist. 1969;9(3):179-86.

15 Hokoishi K, Ikeda M, Maki N, Nomura M, Torikawa S, Fujimoto N, et al. Interrater reliability of the Physical Self-Maintenance Scale and the Instrumental Activities of Daily Living Scale in a variety of health professional representatives. Aging Ment Health. 2001 Feb;5(1):38-40. 
16 Kiadaliri AA, Felson DT, Neogi T, Englund M. Brief Report: Rheumatoid Arthritis as the Underlying Cause of Death in Thirty-One Countries, 1987-2011: Trend Analysis of World Health Organization Mortality Database. Arthritis Rheumatol. 2017 Aug;69(8):1560-5.

17 Saari T, Hallikainen I, Hänninen T, Räty H, Koivisto A. Relationships between Cognition and Activities of Daily Living in Alzheimer's Disease During a 5-Year Follow-Up: ALSOVA Study. J Alzheimers Dis. 2018;64(1):26979.

18 Putcha D, Tremont G. Predictors of independence in instrumental activities of daily living: amnestic versus nonamnestic MCI. J Clin Exp Neuropsychol. 2016 Nov;38(9):991-1004.

19 Barberger-Gateau P, Dartigues JF, Letenneur L. Four Instrumental Activities of Daily Living Score as a predictor of one-year incident dementia. Age Ageing. 1993 Nov;22(6):457-63.

20 Farias ST, Mungas D, Reed BR, Harvey D, Cahn-Weiner D, Decarli C. MCI is associated with deficits in everyday functioning. Alzheimer Dis Assoc Disord. 2006 Oct-Dec;20(4):217-23.

21 Jefferson AL, Barakat LP, Giovannetti T, Paul RH, Glosser G. Object perception impairments predict instrumental activities of daily living dependence in Alzheimer's disease. J Clin Exp Neuropsychol. 2006 Aug;28(6): 884-97.

22 Dubois B, Feldman HH, Jacova C, Hampel H, Molinuevo JL, Blennow K, et al. Advancing research diagnostic criteria for Alzheimer's disease: the IWG-2 criteria. Lancet Neurol. 2014 Jun;13(6):614-29.

23 Kamiya M, Osawa A, Kondo I, Sakurai T. Factors associated with cognitive function that cause a decline in the level of activities of daily living in Alzheimer's disease. Geriatr Gerontol Int. 2018 Jan;18(1):50-6.

24 Jhoo JH, Chi YK, Choi HJ, Han JW, Kim TH, Lee JJ, et al. A normative study of the disability assessment for dementia in community-dwelling elderly koreans. Psychiatry Investig. 2014 Oct;11(4):446-53.

25 Delva F, Edjolo A, Pérès K, Berr C, Barberger-Gateau P, Dartigues JF. Hierarchical structure of the activities of daily living scale in dementia. J Nutr Health Aging. 2014 Jul;18(7):698-704.

26 Kessels RP, Rijken S, Joosten-Weyn Banningh LW, Van Schuylenborgh-VAN Es N, Olde Rikkert MG. Categorical spatial memory in patients with mild cognitive impairment and Alzheimer dementia: positional versus objectlocation recall. J Int Neuropsychol Soc. 2010 Jan;16(1):200-4.

27 Mandal PK, Joshi J, Saharan S. Visuospatial perception: an emerging biomarker for Alzheimer's disease. J Alzheimers Dis. 2012;31(Suppl 3):S117-35.

28 Ikeda Y, Ogawa N, Yoshiura K, Han G, Maruta M, Hotta M, et al. Instrumental Activities of Daily Living: The Processes Involved in and Performance of These Activities by Japanese Community-Dwelling Older Adults with Subjective Memory Complaints. Int J Environ Res Public Health. 2019 Jul;16(14):pii:E2617. 